\section{Maus-tratos contra idosos no município de Camaragibe, Pernambuco}

\section{Elder abuse in Camaragibe, Pernambuco}

Victor Lopes de Melo 1 Juliana de Oliveira Carneiro da Cunha 2 Gilliatt Hanois Falbo Neto 3 1-3 Grupo Estudos da Violência. Diretoria de Pesquisa. Instituto
Materno Infantil Prof. Fernando Figueira. Rua dos Coelhos, 300. Boa Vista. Recife, PE, Brasil. CEP: 50.070-550

\begin{abstract}
Objectives: to describe the situation of abuse against the elderly in the municipality of Camaragibe, in the metropolitan region of Recife, Pernambuco.

Methods: cross sectional study with a representative sample of the elderly population in the municipality, subject to abuse and respondents to a previously designed questionnaire according to the objectives of the study.

Results: of the 315 elderly, 95.4\% were in the sixth and seventh decade of life and $65.2 \%$ were women and received up to two minimum wages $(91.4 \%)$, were house owners $(87.5 \%)$, living in neighborhoods with deficient sewage systems $(84.1 \%)$ and low education level (97.1\%). Seventy six claimed they were abused. Among these $65.2 \%$ were females. The most common type of violence was psychological $(62.1 \%)$, followed by physical abuse $(31.8 \%)$, perpetrated by family members themselves within their own homes. Women were more abused than men. $71.3 \%$ did not react and did not know where to seek help. Their perception is that the community is indifferent to the problem.

Conclusions: education measures could be implemented to clarify the elderly on their rights, to facilitate denouncements and obtainment of support or help.
\end{abstract}

Key words Violence, Aged, Elder abuse

\section{Resumo}

Objetivos: descrever a situação de maus-tratos contra idosos no município de Camaragibe, na região metropolitana do Recife, Pernambuco.

Métodos: estudo descritivo de corte transversal com uma amostra representativa da população idosa do município, e submetida a questionário previamente elaborado conforme os objetivos do estudo.

Resultados: dos 315 idosos, 95,4\% estavam na sexta e sétima década de vida, 65,2\% eram mulheres e recebiam até dois salários mínimos (91,4\%), 87,5\% tinham casas próprias, com deficiente esgotamento sanitário $(84,1 \%)$ e baixo grau de escolaridade $(97,1 \%)$. Sessenta e seis referiram sofrer maus-tratos. Desses, 65,2\% eram do sexo feminino. O tipo de violência mais comum foi a psicológica (62,1\%), seguida da física $(31,8 \%)$, cometidas pelos próprios familiares dentro do próprio domicílio. As mulheres foram mais agredidas que os homens. 71,3\% reagiram passivamente não sabendo a que órgão denunciar. No seu entendimento a comunidade era indiferente ao problema.

Conclusões: os maus-tratos contra idosos apresentam-se como problema relevante para a saúde pública. Medidas educativas poderiam ser implementadas para esclarecê-los sobre direitos, facilitar a denúncia e a obtenção de apoio ou ajuda.

Palavras-chave Violência, Idoso, Maus-tratos ao idoso 


\section{Introdução}

A Assembléia Mundial de Saúde declarou a violência como um problema de saúde pública mundial, tendo em vista suas graves conseqüências a curto e longo prazo para indivíduos, famílias, comunidades e países, além do aumento da demanda que acarreta em serviços de saúde em todo mundo. ${ }^{1}$

As mortes violentas em idosos seriam conseqüência principalmente de acidentes de trânsito e transporte, quedas e homicídios, causas que em conjunto seriam responsáveis por mais de $50 \%$ dos óbitos por causas externas nesse grupo. ${ }^{2}$ As taxas de suicídio, embora se mantenham em um patamar que pode ser considerado baixo, chamam atenção por apresentar taxas mais elevadas na população idosa. ${ }^{3}$ Deve-se considerar, no entanto, que óbitos de idosos muitas vezes atribuídos a causas naturais, acidentais ou desconhecidas, são na verdade conseqüência de abuso ou negligência. 4

Os maus-tratos contra idosos foram descritos pela primeira vez em 1975, em publicações britânicas, e desde então têm sido tema de pesquisas científicas e alvo de ações governamentais em todo mundo, e no Brasil, especificamente, desde a última década. 4 Com o envelhecimento da população mundial, esse tema vem adquirindo mais e mais relevância. De acordo com a Wolf et al.,4 o número de idosos em todo o mundo deve chegar a cerca de 1,2 bilhões, em 2025 , e representar $12 \%$ da população dos países em desenvolvimento.

A violência contra o idoso pode assumir várias formas e ocorrer em diferentes situações; e por diferentes motivos é subdiagnosticada e subnotificada. 5 Segundo Cammer Paris, 6 entre as causas para o difícil diagnóstico estão: sentimentos da vítima de culpa e vergonha, medo de retaliação ou represália por parte do agressor, ou ainda receio de ser internada em asilo. A maioria dos casos de violência contra idosos é devido à auto-negligência ou é perpetrado por um membro da família, 7 o que pode explicar porque as vítimas tendem a minimizar a gravidade da agressão e se mostrarem leais a seu agressor, freqüentemente negando-se a adotar medidas legais contra membros da família ou a discutir sobre esse assunto com terceiros. 6,7 Elas preferem conviver com maus-tratos a abrir mão de um relacionamento pessoal em suas vidas. ${ }^{6}$

Um estudo realizado por Krueger e Patterson 8 procurou mostrar as dificuldades apontadas pelos médicos para a detecção de violência contra o idoso. Entre os principais problemas estavam: negação do paciente e/ou da família de que existe o mau-trato; oposição à intervenção, uma vez identificado o abuso; falta de conhecimento sobre a definição e prevalência de violência contra o idoso; inexistência de uma agência à qual se possa denunciar o mautrato; e falta de um consenso sobre diagnóstico e conduta diante de violência contra o idoso.

Vários estudos foram realizados na tentativa de determinar quais os fatores associados a maus-tratos contra idosos. História de abuso de álcool ou drogas e de distúrbio psicopatológico no cuidador $2,4,5,9$ ou em sua família ${ }^{6}$ são fatores de risco bem conhecidos. Diminuição de capacidade cognitiva e física e conseqüentemente uma maior dependência, foi consideda inicialmente como fator de risco para a violência contra idosos; $2,4,6$ entretanto estudos posteriores mostraram que idosos maltratados não eram mais debilitados que aqueles que não sofriam maus-tratos, sendo talvez até mais capazes, especialmente nos casos de abuso físico e verbal. ${ }^{4}$ Os casos de maustratos comunicados às autoridades, contudo, envolvem mais freqüentemente os mais idosos e dependentes. 4 O nível de estresse do cuidador também foi inicialmente considerado um relevante fator de risco para maus-tratos contra idosos, 6 e embora ainda se acredite que seja um fator contribuinte para a ocorrência de abuso, ele não responde, por si só, pela ocorrência do mesmo. 4

Um comportamento violento ou agressivo do idoso é uma situação de risco, 2 e pode provocar violência recíproca por parte do cuidador, 4 mesmo que não se observe mais esse comportamento violento no presente, de forma que também se considera a história de violência familiar transgeracional como um importante fator de risco.2,4-6 Recentemente, estudos têm reforçado a idéia de que o relacionamento entre o idoso e o cuidador, antes de estabelecida a dependência, seria mais relevante até do que o nível dessa dependência ou o grau de estresse do cuidador.4,9

As condições de vida devem ser consideradas um fator de risco por serem causa de conflitos familiares, ${ }^{4-6}$ particularmente a aglomeração e a falta de privacidade; 4 e ainda que a violência contra o idoso possa ocorrer com a vítima e o agressor vivendo separadamente, o risco é maior quando o perpetrador vive na mesma casa que o idoso. 2,4

Tanto o idoso sustentado por seu cuidador quanto um cuidador financeiramente dependente de um idoso mostram-se como situações de risco.2,4 Parece haver uma rede de interdependência que frustra os esforços de intervenção nessa situação. ${ }^{4}$

$\mathrm{O}$ isolamento social é fator de risco para maustratos contra idosos, podendo se apresentar como causa2,6 ou conseqüência nas vítimas de abuso, 4 além de ser considerado um fator causal quando 
observado em agressores. 2,5,6

O grupo dos idosos vitimados por maus-tratos apresenta também uma taxa de mortalidade muito mais alta que o dos idosos que não sofreram abuso, mesmo quando eliminados os fatores confundidores. 4

Apesar da relevância do tema para a saúde pública, observamos uma escassez de estudos no nosso meio. Reveste-se, portanto de importância a tentativa de descrever a situação de maus-tratos contra idosos no município de Camaragibe, Pernambuco, pela oportunidade que propicia a produção de novos conhecimentos e de oferecer subsídios para a formulação de políticas públicas de proteção e promoção à saúde desse grupo populacional de risco.

\section{Métodos}

O projeto se desenvolveu no município de Camaragibe, situado na Região Metropolitana do Recife (RMR) - no Estado de Pernambuco, Nordeste do Brasil - com uma população de 143.732 habitantes, 10 e densidade demográfica de $2.100 \mathrm{hab} / \mathrm{km}^{2}$. O Programa de Saúde da Família (PSF) no município conta com 32 unidades que atendem a 32.256 famílias, o correspondente a $92 \%$ da população. O Programa de Agentes Comunitários de Saúde (PACS) tem 231 integrantes, atuando junto com as equipes do PSF.

A população do estudo foi uma amostra representativa para o município da população de adultos idosos (60 anos ou mais), recrutada na Unidade de Saúde da Família (USF) do bairro de Céu Azul.

O perfil da população estudada foi traçado através do levantamento estatístico de alguns indicadores considerados de referência na avaliação da saúde do idoso.

O instrumento de coleta de dados constou de formulários com questões para atender aos objetivos previamente fixados. Para fins comparativos com alguns indicadores, na elaboração do instrumento de coleta de dados foi utilizado como referencial o questionário da II Pesquisa Estadual de Saúde e Nutrição, Pernambuco de 1997, base de dados mais recente do Estado, tendo sido realizadas ainda ampliações para cobrir objetivos do trabalho não contemplados na referida pesquisa. Os dados do questionário usados na análise incluíam identificação, registro domiciliar, avaliação socioeconômica, rastreamento geronto-clínico e violência contra o idoso. O questionário sobre violência, especificamente, atendia aos seguintes objetivos: a) quantificar o número de idosos vítimas de maustratos; b) quantificar e qualificar os tipos de maustratos dos quais os idosos eram vítimas; c) determinar o local onde ocorreram os maus-tratos; d) identificar o perpetrador dos maus-tratos; e) determinar a reação dos idosos maltratados frente ao abuso; f) determinar se os idosos sabam a que órgão deveriam denunciar os maus-tratos; g) determinar se os idosos tinham conhecimento sobre a existência de algum idoso vítima de maus-tratos; h) determinar a reação da comunidade frente a um caso de maustratos contra idosos; i) determinar se os idosos tinham conhecimento da existência de alguma instituição de apoio na comunidade e identifica-la.

$\mathrm{O}$ questionário foi testado num estudo piloto envolvendo $10 \%$ da população atendida pela unidade do PSF selecionada para o estudo, e foi aplicado por pesquisadores do Instituto de Nutrição da Universidade Federal de Pernambuco e do Instituto Materno Infantil Prof. Fernando Figueira (IMIP), com o apoio da equipe do PSF e dos PACS, após treinamento prévio.

Para a inserção do idoso na pesquisa, o termo de consentimento livre e esclarecido deveria ser assinado pelo idoso e/ou familiar responsável.

Para análise e processamento dos dados foi utilizado o programa estatístico Epi-info, versão 6.04d. Os dados foram digitados com dupla entrada e verificadas a sua consistência e validação. Para as análises estatísticas e testes de associação, foi utilizado o qui-quadrado (Yates) e Fisher adotandose um intervalo de confiança de $95 \%$, e um valor de $p<0,05$ para rejeição de hipótese nula.

O estudo foi pautado pelas normas do Comissão Nacional de Ética em Pesquisa do Conselho Nacional de Saúde, contidas da resolução 196/96 e foi submetido ao Comitê de Ética em Pesquisa da Universidade Federal de Pernambuco.

\section{Resultados}

Dos 315 sujeitos pesquisados, 291 (90,8\%) estavam na sexta e sétima décadas de vida. O sexo feminino foi preponderante, com 196 (62,2\%) dos idosos.

Dos 315 idosos estudados, 310 responderam quando perguntados se haviam sofrido maus-tratos, e $66(21 \%)$ responderam que sim.

$\mathrm{Na}$ Tabela 1, observamos a porcentagem de homens e mulheres que referem ter sido vítimas de violência.

Uma renda de até um salário mínimo foi observada em 191 (78,3\%) dos casos. Dos idosos estudados, $278(97,9 \%)$ moravam em casa; e dessas, 246 
Tabela 1

Sexo e maus-tratos

\begin{tabular}{lrrrrr}
\hline Sexo & \multicolumn{5}{c}{ Maus tratos } \\
& \multicolumn{2}{c}{ Sim } & \multicolumn{2}{c}{ Não } & Total \\
\cline { 2 - 6 } & \multicolumn{1}{c}{$\mathrm{n}$} & $\%$ & $\mathrm{n}$ & $\%$ & \\
\hline Masculino & 23 & 34,8 & 95 & 38,9 & 118 \\
Feminino & 43 & 65,2 & 149 & 61,1 & 192 \\
Total & 66 & 100,0 & 244 & 100,0 & 310 \\
\hline
\end{tabular}

$p=0,643$

Tabela 2

Sexo x tipo de maus-tratos.

\begin{tabular}{lrrrrr}
\hline Tipo de mau-trato & \multicolumn{5}{c}{ Sexo } \\
& \multicolumn{2}{c}{ Masculino } & \multicolumn{2}{c}{ Feminino } & Total \\
\cline { 2 - 6 } & \multicolumn{1}{c}{$\mathrm{n}$} & $\%$ & $\mathrm{n}$ & $\%$ & \\
\hline Físico & 4 & 17,4 & 17 & 40,0 & 21 \\
Psicológico & 17 & 73,9 & 24 & 55,6 & 41 \\
Negligência & 2 & 8,7 & 2 & 4,4 & 4 \\
Total & 23 & 100,0 & 43 & 100,0 & 66 \\
\hline
\end{tabular}

$p=0,078$ (mau-trato físico $\times$ mau-trato pscicológico)

Tabela 3

\begin{tabular}{|c|c|c|c|c|}
\hline \multirow[t]{3}{*}{ Sexo } & \multicolumn{4}{|c|}{ Local onde ocorreram os maus-tratos } \\
\hline & \multicolumn{2}{|c|}{ Na comunidade } & \multicolumn{2}{|c|}{$\begin{array}{c}\text { Dentro de casa } \\
\text { (na família) }\end{array}$} \\
\hline & $\mathrm{n}$ & $\%$ & $\mathrm{n}$ & $\%$ \\
\hline Masculino & 10 & 52,6 & 9 & 22,0 \\
\hline Feminino & 9 & 47,4 & 32 & 78,0 \\
\hline Total & 19 & 100,0 & 41 & 100,0 \\
\hline
\end{tabular}

$p=0,038$
$(87,5 \%)$ eram próprias. Apenas $45(15,9 \%)$ dessas moradias eram ligadas à rede de esgotos. Duzentas e catorze $(76,5 \%)$ tinham fossas sépticas, e $24(8,5 \%)$ não tinham esgotamento sanitário algum.

Quanto à escolaridade, foi evidenciado que 123 $(44,1 \%)$ idosos não haviam concluído o primeiro grau, e 148 (53\%) não haviam concluído o segundo.

A residência foi o local onde mais freqüentemente ocorreram os maus-tratos, com $41(62,1 \%)$ relatos de violência. Os principais perpetradores desses maus-tratos foram: familiares, em $46(69,8 \%)$ ocasiões; vizinhos, em $8(12,1 \%)$; desconhecidos, em 5 (7,6\%); e outros em 4 (6\%) casos.

Quando perguntados sobre como se portaram diante do fato, os idosos agredidos responderam: 47 $(71,3 \%)$ não reagiram; $13(19,7 \%)$ reagiram; e 3 $(4,5 \%)$ denunciaram às autoridades.

Dos 315 idosos pesquisados, 146 (46,3\%) expressaram que sabiam a qual órgão público poderiam denunciar a agressão, no entanto nenhum deles foi capaz de nominar um órgão de defesa dos idosos. Quando indagados se tinham conhecimento de algum caso de maus-tratos a um idoso na comunidade, apenas $24(7,6 \%)$ responderam afirmativamente.

Quando perguntados como a comunidade reagia ante casos de maus-tratos contra idosos, $161(51,1 \%)$ não responderam; 85 (27\%) afirmaram que a comunidade desaprovava e tomava alguma providência; e $69(21,9 \%)$, que a comunidade não tomava qualquer atitude.

Apenas 67 (21,3\%) responderam que tinham conhecimento de alguma instituição de apoio ao idoso no município. Entre as instituições citadas, apareceram grupos da terceira idade, asilos e albergues, além de outras instituições.

Ao analisarmos sexo e o tipo de maus-tratos (Tabela 2) a que foram submetidos os idosos, verificamos que houve $41(62,1 \%)$ casos de violência psicológica, $21(31,8 \%)$ de violência física, e 4 $(6,1 \%)$ casos de negligência. Nenhum dos entrevistados referiu ter sido vítima de violência sexual. Das vítimas de violência física, 17 eram do sexo feminino, e 4 eram do sexo masculino. O abuso psicológico vitimou 24 mulheres, que representam $58,5 \%$ do total de idosos acometidos por esse tipo de mau-trato. Já os casos de negligência, foram relatados por 2 homens e 2 mulheres.

Em relação ao local onde ocorreu o mau-trato (Tabela 3), constatamos que das agressões cometidas na comunidade, $52,6 \%$ foram contra homens e $47,4 \%$, contra mulheres. Enquanto que em seus próprios domicílios, as mulheres foram mais agredidas $(78 \%)$ que os homens $(22 \%)(p<0,038)$. Uma 
análise considerando apenas os maus-tratos físicos e psicológicos apresentou proporções diferentes, que embora não significantes $(p=0,078)$, indicam uma possível tendência associada ao gênero.

Quanto ao tipo de convivência, $41(68,3 \%)$ idosos maltratados relataram uma convivência multigeracional, e 19 (31,7\%) referiram convivência unigeracional. Dos entrevistados não-maltratados, 164 $(79,2 \%)$ afirmaram viver em ambiente multigeracional, e $43(20,8 \%)$ conviviam com pessoas da mesma geração.

Dos idosos que relataram maus-tratos, 40 $(66,7 \%)$ disseram estar envolvidos nas atividades familiares, enquanto $20(33,3 \%)$ não participavam de tais atividades. Entre os que negaram ser vítimas de abuso, $163(76,5 \%)$ faziam parte de atividades em família, e $50(23,5 \%)$, se abstinham.

A participação em associações comunitárias foi referida por $14(23,3 \%)$ dos idosos que sofriam maus-tratos, e negada por $46(76,6 \%)$ deles. Quarenta e sete $(22,1 \%)$ entrevistados que não foram maltratados eram membros dessas associações, e 166 $(77,9 \%)$ negaram participação em instituições do tipo.

\section{Discussão}

$O$ fato de não haver diferença entre homens e mulheres que referiram ter sido vítimas de violência e o número total de homens e mulheres entrevistados, evidenciou que a prevalência de abuso contra o idoso não distinguiu sexos no grupo estudado. Esse resultado, apesar de estar de acordo com estudos realizados no Canadá, Países Baixos e EUA, ${ }^{4}$ vai de encontro aos achados de pesquisas na Finlândia 4 e dados do National Aging Resource Center, nos EUA, ${ }^{11}$ nos quais se observa uma maior prevalência de maus-tratos em idosos do sexo feminino. A maior ocorrência de violência psicológica contradiz achados da literatura pesquisada, que evidencia o abuso físico como o tipo mais comum de violência contra o idoso. ${ }^{5} \mathrm{O}$ achado onde os homens relataram maior percentual de abuso psicológico que as mulheres pode advir do fato de que, quando vítimas de abuso emocional, eles denunciam com mais freqüência que as mulheres. ${ }^{4}$ Vale ressaltar que o número absoluto de mulheres vítimas de violência física foi aproximadamente quatro vezes maior que o número de homens.

Neste estudo nenhum dos entrevistados referiu ter sido vítima de violência sexual, assim como poucos estudos abordam esse tema de forma específica, com informações epidemiológicas.
Teaster et al. 12 e Roberto e Teaster 13 estudando abuso sexual em 42 idosos evidenciaram que a maioria dos casos de violência sexual contra idosos envolve mulheres, desorientadas em dois de três domínios (tempo, espaço, pessoa), vivendo em asilos, agredidas mais freqüentemente por outros residentes do asilo. Os mesmos autores afirmam que há uma maior tendência de mulheres com dificuldade de deambulação serem vítimas de mais de um tipo de violência sexual do que as mulheres que não precisam de auxílio para deambular. Os tipos de abuso mais cometidos eram beijos e atos sexuais não consentidos e interesse sexual no corpo da mulher. ${ }^{14}$

No presente estudo a maioria dos perpetradores tinha 60 anos ou mais. As mulheres que viviam na comunidade eram mais agredidas por membros da família, enquanto as que viviam em asilos, eram vítimas de outros residentes. 13

Em sua pesquisa, Minayo 2 afirma que é mais comum a vitimização do sexo feminino pela violência intra-domiciliar e que a violência urbana incide mais freqüentemente sobre os homens. Observamos que, de fato, o número de mulheres agredidas dentro de casa foi quase quatro vezes maior que o número de homens, diferentemente da violência sofrida na comunidade. $\mathrm{O}$ abuso contra idosos parece ser de fato, perpetrado mais freqüentemente por um membro da família, como afirmam Minayo $^{2}$ e Harrell et al. ${ }^{7}$, visto que o abuso ocorre mais freqüentemente no domicílio.

Apesar da possibilidade de que conflitos transgeracionais levem a abuso contra idosos, ${ }^{2}$ não houve diferença na prevalência de maus-tratos nos idosos que viviam num ambiente uni ou multigeracional.

A participação de idosos em associações comunitárias também não modificou a prevalência de abuso, não confirmando, no grupo estudado, a idéia de que o isolamento da vítima seria um fator associado. ${ }^{6}$ Não foi possível avaliar se o isolamento seria uma barreira ao diagnóstico dos maus-tratos; ${ }^{7}$ no nosso caso o diagnóstico foi baseado na resposta do entrevistado. Por esse motivo, pode-se inferir que um maior número de casos pode não ter sido detectado por omissão na resposta. 6 No entanto, um percentual considerável $(21 \%)$ dos idosos referiu maus-tratos, embora nenhum soubesse a que órgão denunciar. Mais de 20\% dos entrevistados consideravam que a comunidade não tomaria atitude alguma caso ficasse ciente de um caso de maus-tratos contra idosos. Poucos entrevistados conheciam alguma instituição de apoio ao idoso no município.

Os maus-tratos contra idosos no município apresentam-se como um problema relevante para saúde pública, pois atingem cerca de um quinto desse 
grupo populacional. Medidas educativas e de mobilização social poderiam ser implementadas com o objetivo de esclarecer a esse grupo os seus direitos, orientar quanto à atitude apropriada a adotar diante da agressão, facilitar a denúncia e a obtenção de apoio ou ajuda, buscar junto a sociedade uma sensibilização para o problema e sua participação na construção de uma rede de proteção ao idoso. Essa que poderia envolver o sistema de saúde, pelo poder executivo, com os agentes comunitários de saúde e as equipes de saúde da família, os órgãos da segu-

\section{Referências}

1. WHA (World Health Assembly). Prevention of violence: a public health priority: forty-ninth. 3. ed. Geneva; 1996.

2. Minayo MC. Violência contra idosos: relevância para um velho problema. Cad Saúde Pública. 2003; 19: 783-91.

3. Mello Jorge MH, Gawryszewski VP, Latorre MR. I. Análise dos dados de mortalidade. Rev Saúde Pública. 1997; 31: 525.

4. Wolf R, Daichmann L, Bennett G. Abuse of the elderly. In: Krug Eg, Dahlberg LL, Nercy JA, ZW, AB, Lozano R, editors. World report on violence and health. Geneva: World Health Organization; 2002. p. 123-45

5. Bradley M. Elder abuse. Br Med J. 1996; 313: 548-50.

6. Cammer Paris BE. Violence against elderly people. Mt Sinai J Med. 1996; 63: 97-100.

7. Harrell R, Toronjo CH, McLaughlin J, Pavlik VN, Hyman DJ, Dyer CB. How geriatricians identify elder abuse and neglect. Am J Med Sci. 2002; 323: 34-8.

8. Krueger P, Patterson C, The Research Subcommittee of the Elder Abuse and Self-Neglect Task Force of HamiltonWentworth. Detecting and managing elder abuse: challenges in primary care. CMAJ. 1997; 157: 1095-100. rança pública, os órgãos do poder judiciário (ministério público), o poder legislativo municipal e a sociedade civil por meio das suas organizações.

\section{Agradecimentos}

Ao Instituto Materno Infantil Prof. Fernando Figueira, (IMIP) pelo suporte e ao Conselho Nacional de Desenvolvimento Científico e Tecnológico, CNPQ pela bolsa do Programa de Iniciação Científica.
9. MacLennan W. Abuse of the elderly: chapter in the World Report on Violence. Age Ageing. 2003; 32: 136.

10. IBGE (Instituto Brasileiro de Geografia e Estatística). Estimativas de população para $1^{\circ}$ de julho de 2004. Disponível em: http://www.ibge.gov.br/ (2005 jun 27).

11. Drake V, Freed PE. Research applications: domestic violence in the elderly. Geriatr Nurs. 1998; 19: 165-7.

12. Teaster PB, Roberto KA, Duke JO, Kim M. Sexual abuse of older adults: preliminary findings of cases in Virginia. J Elder Abuse Neglect. 2000; 12: 17-51.

13. Roberto KA, Teaster PB. Sexual abuse of vulnerable young and old women: a comparative analysis of circumstances and outcomes. Violence Against Women. 2005; 11: 473504.

14. Teaster PB, Roberto KA. Sexual abuse of older women living in nursing homes. J Gerontol Soc Work. 2003; 4: 105-37.

Recebido em 28 de setembro de 2005

Versão final apresentada em 25 de janeiro de 2006

Aprovado em 13 de janeiro de 2006 